\title{
Tendinopatía cálcica: Etiopatogenia y evaluación por imágenes
}

\author{
Nicolás García A. ${ }^{\star}$, Julio Rosales L.', Marco Antonio Verdugo P.'
}

1. Radiólogo, Unidad de Imágenes, Clínica MEDS. Santiago, Chile.

\section{Calcific tendinitis: Aetiopathogenesis and imaging evaluation}

\begin{abstract}
Resumen:
La tendinopatía cálcica es causada por el depósito patológico de cristales de hidroxiapatita de calcio en los tendones y es una causa común de dolor en las articulaciones. Afecta más frecuentemente al hombro y la cadera, con hallazgos característicos en imágenes; sin embargo, cualquier tendón puede estar involucrado. Ocasionalmente, la tendinopatía cálcica puede simular patología agresiva, como infección o neoplasia, especialmente en RM. Fisiotpatológicamente, las calcificaciones provendrían de una diferenciación anormal de las células madre del tendón, que comienzan a producir calcio, aunque todavía no es del todo claro. Los radiólogos deben estar familiarizados con los hallazgos de las imágenes para distinguir la tendinopatía cálcica de procesos más agresivos. La aspiración y lavado guiado bajo ecografía es una técnica útil realizada por el radiólogo para el tratamiento de casos sintomáticos. La familiaridad con estos procedimientos y su apariencia en imágenes es un aspecto importante en el manejo de esta enfermedad. El propósito de esta revisión es analizar la etiopatogenia de la tendinopatía cálcica, la evaluación con imágenes en los sitios de presentación más comunes y también en los menos frecuentes, así como el papel que desempeña la ecografía en el tratamiento de la patología.
\end{abstract}

Palabras clave: Hidroxiapatita de calcio; Manguito rotador; Tendinitis; Tendinopatía cálcica.

\begin{abstract}
:
Calcific tendinitis is caused by abnormal deposition of calcium hydroxyapatite crystals in tendons and is a common cause of joint pain. The disease typically affects the shoulder and hip, with characteristic imaging findings; however, any tendon can be involved. Occasionally, calcific tendinitis can mimic aggressive disorders, such as infection and neoplasm, especially on MRI. Apparently, the calcifications come from an abnormal differentiation of the tendon stem cells, which begin to produce calcium.

Radiologists should be familiar with the imaging findings to distinguish calcific tendinitis from more aggressive processes. Image-guided percutaneous needle aspiration is a useful technique performed by the radiologist for the treatment of symptomatic cases. Being familiar with these processes and their imaging appearance is an important aspect in the management of this common disease. The purpose of this review is to analyze the pathogenesis of calcium tendinopathy, the evaluation of images in both the most common and less frequent presentation sites, as well as the role played by ultrasound in the treatment of pathology. Keywords: Calcium hydroxyapatite disease; Calcific tendinitis; Rotator cuff; Tendinitis; Tendinopathy.
\end{abstract}

García A., et al. Tendinopatía cálcica: Etiopatogenia y evaluación por imágenes. Rev Chil Radiol 2020; 26 (2): 52-61. ${ }^{*}$ Correo electrónico: Nicolás García A. / nicolasgarciaa@gmail.com

Trabajo enviado el 28 de enero de 2020. Aceptado para publicación el 04 de mayo de 2020.

\section{Introducción}

La tendinopatía cálcica es el depósito de cristales de hidroxiapatita cálcica en los tendones. Fue descrita por primera vez por Codman en los tendones del manguito rotador ${ }^{(1)}$. Clínicamente también se conoce como tendinitis cálcica. Puede afectar prácticamente cualquier tendón, en general cercano a su inserción ósea, siendo el hombro por lejos el sitio más frecuente, seguido de la cadera ${ }^{(2)}$. En el hombro, donde existen mayor cantidad de estudios epidemiológicos, la frecuencia es variable según la serie evaluada, con incidencias reportadas entre un 
3-8\% en pacientes asintomáticos y entre un $33-42 \%$ en pacientes con hombro doloroso, siendo bilateral en el 10 a $20 \%$ de los $\operatorname{casos}^{(3,4,5)}$. La edad más frecuente en que se presenta es entre los 30 y 60 años, siendo levemente más prevalente en mujeres ${ }^{(6)}$. El tendón del supraespinoso es el más frecuentemente afectado (aproximadamente el $50 \%$ de los casos), seguido del infraespinoso (40\%), siendo el subescapular y redondo menor mucho menos prevalentes ${ }^{(4)}$.

Clínicamente, la tendinopatía cálcica se presenta en 3 posibles escenarios: hallazgo incidental sin síntomas, como dolor crónico de bajo grado y presentación aguda muy dolorosa con marcada limitación funcional.

La presente revisión tiene por objetivo analizar la etiopatogenia de la tendinopatía cálcica, la evaluación por imágenes tanto en los sitios más comunes de presentación como en los menos frecuentes, así como también el papel que juega la ecografía en el tratamiento de la patología.

\section{Etiopatogenia}

La fisiopatología de la tendinopatía cálcica está lejos de ser completamente comprendida. Varias teorías se han descrito acerca del origen de los depósitos de cristales de hidroxiapatita. Codman ${ }^{(1)}$ hipotetizó que el sobreuso y la degeneración tendínea tenía que ver en la fisiopatología de la tendinopatía cálcica. Otras publicaciones fueron en la misma línea, como la de Wolfang ${ }^{(7)}$, que en 1974 publicó que en pacientes operados por rotura del manguito rotador el $23 \%$ tenía calcificaciones; Jim y cols. ${ }^{(8)}$ en 1993 postuló a la degeneración tendínea como una de las causas de las calcificaciones, basado en la observación que el $28 \%$ de los pacientes con tendinopatía cálcica tenía además rotura tendínea, y que ambas se producen en la zona crítica del tendón, argumentando que la degeneración del tendón produce necrosis focal, la que finalmente se traduce en calcificación. Sin embargo, varias observaciones más recientes han ido contradiciendo tal idea. Por ejemplo, el peak de edad de la tendinopatía cálcica es menor que el de patología degenerativa; además, se han descrito casos de tendinopatía cálcica en prácticamente todos los rangos de edad, incluso en niños ${ }^{(9,10)}$. Por otro lado, la tendinopatía cálcica se resuelve ad-integrum ${ }^{(3)}$, mientras que la patología degenerativa no. En cuanto a su relación con las roturas, en un estudio reciente de Beckmann y cols. ${ }^{(11)}$ se evaluaron 86 pacientes con tendinopatía cálcica en RM comparados con un grupo control pareado por edad, género y lateralidad, encontrando que no habían diferencias estadísticamente significativas en la incidencia de roturas en ambos grupos. Anteriormente, también se habían publicado algunas series de pacientes jóvenes con tendinopatía cálcica sin incidencia de roturas ${ }^{(12)}$.

Es necesario aclarar que dentro de la patología degenerativa sí se puede encontrar calcificaciones distróficas (las comúnmente llamadas "calcificaciones entesopáticas"). Sin embargo, este tipo de calcificaciones tienen una composición química completamente distinta a los depósitos de hidroxiapatita de calcio de la tendinopatía cálcica, encontrando múltiples sales de calcio (pirofosfato, hidroxiapatita, carbonato, oxalato), así como también diferentes proporciones calcio/fósforo ${ }^{(13,14)}$. Además, su disposición es lineal, adyacente a la inserción ósea del tendón.

La teoría acerca de la etiopatogenia que tiene mayor sustento en la comunidad científica es la planteada por Uhthoff y cols. ${ }^{(14,15)}$, en donde asocia un mecanismo de metaplasia en la cual las células tendíneas se transforman a fibrocartílago para finalmente calcificarse. Al parecer, se trataría de una diferenciación anómala de células madres derivadas de tendón ${ }^{(16)}$. En esta línea, se han descrito diferentes etapas en su evolución, las que en total duran entre 6 a 10 semanas, aunque en algunos casos puede extenderse por varias semanas más ${ }^{(17)}$ :

- Pre-cálcica: Metaplasia fibrocartilaginosa en el tendón, raramente sintomática.

- Cálcica: Se subdivide en:

- Formativa: Transformación fibrocartilaginosa, depósito tipo "tiza".

- Reposo: Depósitos cálcicos duros, fase "silenciosa" o "mecánica".

- Reabsortiva: Depósito con apariencia de "pasta de dientes", puede migrar (ej, a bursas). En esta etapa, se produce una gran reacción inflamatoria asociado a un proceso de neovascularización y neoinervación en el tendón, lo que se traduce clínicamente en mucho dolor ${ }^{(18)}$.

- Post-cálcica: Restauración del patrón normal del tendón.

Sin embargo, todavía quedan múltiples interrogantes por resolver. No está del todo claro lo que determina a las células tendíneas iniciar el proceso de metaplasia a fibrocartílago; tampoco se ha resuelto el por qué se inicia la fase reabsortiva, donde se manifiesta clínicamente la patología. Se ha investigado la posible asociación de patologías sistémicas con la tendinopatía cálcica, sin embargo, hasta ahora sólo se tratan de trabajos descriptivos o en grupos muy específicos, en donde describen cierta mayor frecuencia de tendinopatía cálcica en pacientes con algunos desórdenes endocrinos ${ }^{(19,20)}$, pero que debido a su metodología, no son suficientes para establecer una asociación causal real.

\section{Evaluación por imágenes Radiografía simple}

La radiografía nos permite evaluar la localización, la morfología, el tamaño y la densidad de las calcificaciones (Figura 1). En el hombro, las proyecciones más útiles para tales efectos son la anteroposterior, outlet 


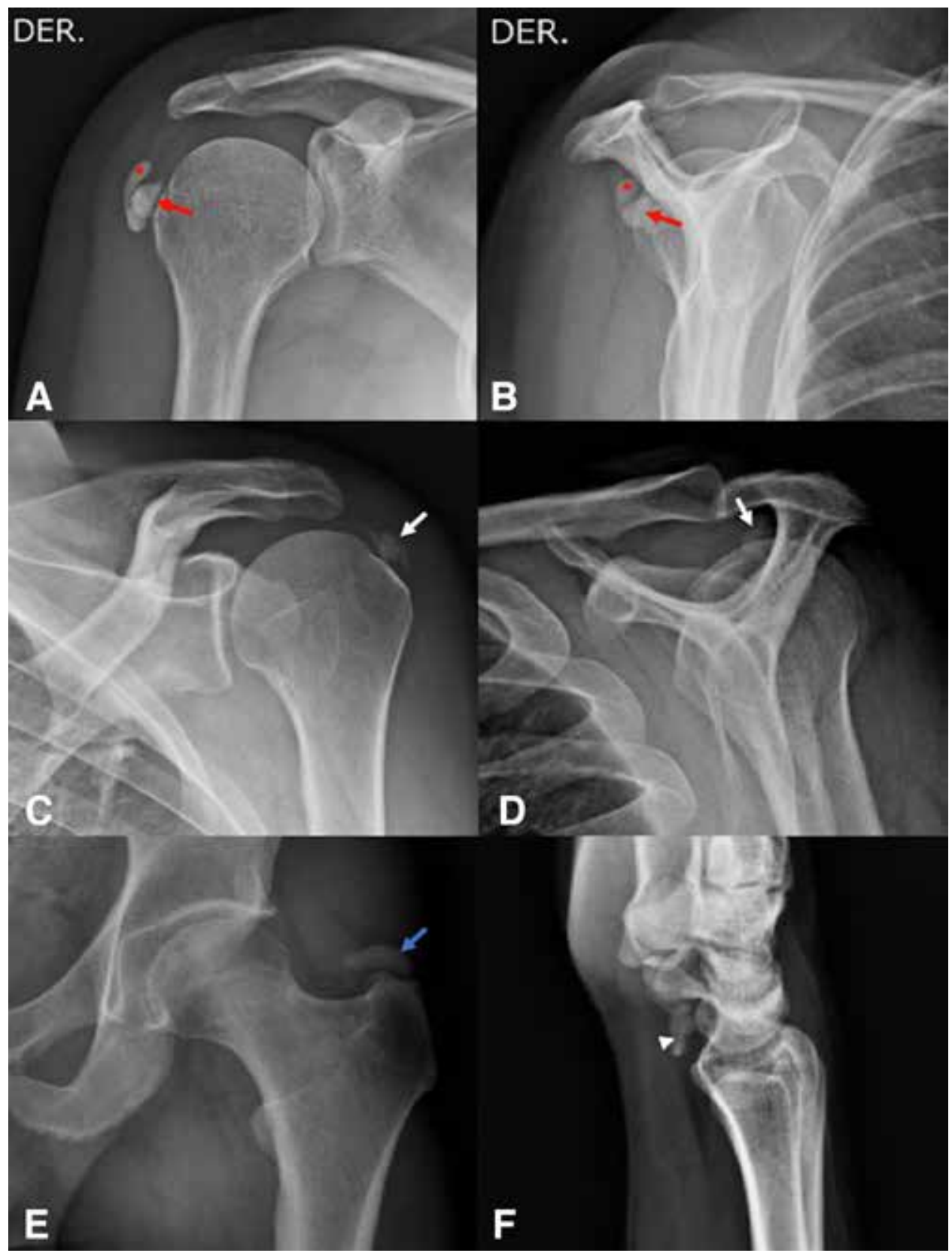

Figura 1: Distintas formas de presentación en diferentes localizaciones de calcificaciones en radiografía. $A, B$. Proyecciones AP y outlet de hombro muestra una calcificación bien definida (flecha roja) en el sitio de proyección del infraespinoso, con un segundo componente $\left(^{*}\right)$ posiblemente en la bursa subacromial subdeltoidea. $C, D$. Proyecciones AP y outlet de hombro de otro paciente muestra una calcificación algo más heterogénea (flecha blanca) que la de $A$ y $B$, en el sitio de proyección del supraespinoso. E. Calcificación bien definida (flecha azul) en el glúteo medio. F. Calcificación heterogénea en el sitio de proyección de los flexores de la muñeca (cabeza de flecha).

y las anteroposterior con rotación interna y externa.

Muchos autores han intentado clasificar radiológicamente las calcificaciones según su tamaño o morfología (Tabla 1). Sin embargo, el hecho de que existan varias clasificaciones es un indicativo de que no existe aquella que correlacione perfectamente el hallazgo radiológico con el clínico, además de tener una alta variabilidad interobservador ${ }^{(5,21)}$.

\section{Ultrasonido}

La ecografía juega un rol fundamental en el diagnóstico de la tendinopatía cálcica. Permite la caracterización de las calcificaciones, incluyendo la forma, tamaño y morfología. A diferencia de la radiografía, la ecografía tiene mejor correlación con las fases de la tendinopatía cálcica, y por ende mejor correlación con la sintomatología ${ }^{(22,23,24)}$. En 
Tabla 1. Clasificaciones radiográficas para la tendinopatía cálcica del manguito rotador.

\begin{tabular}{|c|c|c|}
\hline Autor & Subtipo & Descripción \\
\hline Bosworth (1941) (3) & $\begin{array}{l}\text { Pequeña } \\
\text { Mediana } \\
\text { Grande }\end{array}$ & $\begin{array}{l}<0,5 \mathrm{~cm} \\
0,5-1,5 \mathrm{~cm} \\
>1,5 \mathrm{~cm}\end{array}$ \\
\hline DePalma y cols. $(1961)^{(4)}$ & $\begin{array}{l}\text { Tipo I } \\
\text { Tipo II }\end{array}$ & $\begin{array}{l}\text { Algodonosa, amorfa, mal definida } \\
\text { Homogénea, bien definida }\end{array}$ \\
\hline $\begin{array}{l}\text { Molè y cols. (Asociación Francesa } \\
\text { de Artroscopía) }(2008)^{(21)}\end{array}$ & $\begin{array}{l}\text { Tipo A } \\
\text { Tipo B } \\
\text { Tipo C } \\
\text { Tipo D }\end{array}$ & $\begin{array}{l}\text { Densa, redondeada, bien definida } \\
\text { Multilobulada, bien definida } \\
\text { Heterogénea, irregular } \\
\text { Calcificación distrófica (entesopática) }\end{array}$ \\
\hline Gartner y cols. $(1990)^{(24)}$ & $\begin{array}{l}\text { Tipo I } \\
\text { Tipo II } \\
\text { Tipo III }\end{array}$ & $\begin{array}{l}\text { Bien definida, densa } \\
\text { Densidad intermedia, contornos suaves } \\
\text { Mal definida, baja densidad }\end{array}$ \\
\hline
\end{tabular}

la fase de reposo, las calcificaciones presentan un arco ecogénico bien definido asociado a sombra acústica posterior (Figura 2a), mientras que en la fase reabsortiva se presentan menos definidas, con morfología polimorfa (puntiformes, nodulares e incluso quísticas), con sombra acústica parcial o ausente (Figuras 2b, 2c). El Doppler también puede ser de utilidad, demostrando mayor vascularización en la fase reabsortiva ${ }^{(25)}$ (Figura 3).

\section{Tomografía computada}

La tomografía computada (TC) no es el examen de elección para la tendinopatía cálcica, en general se trata de hallazgos incidentales en evaluación de un hombro doloroso. En la TC, las calcificaciones tienen una apariencia similar que, en la radiografía, encontrando desde calcificaciones homogéneas y bien definidas hasta heterogéneas e incluso puntiformes (Figura 4). En localizaciones menos frecuentes, la TC puede ayudar al diagnóstico al localizar la calcificación en el tendón. También es de gran ayuda en los casos donde hay algunos hallazgos que podrían hacer sospechar una lesión agresiva, como erosión ósea ${ }^{(26)}$. En estos casos, las calcificaciones en el trayecto del tendón es una de las claves diagnósticas (calcificaciones "en cola de cometa")(27).

Figura 2: Diferentes presentaciones ecográficas de las calcificaciones intratendíneas. A. Imagen nodular bien definida, con sombra acústica posterior, se asocia a la fase de reposo. B. Calcificación de aspecto "blanda", sin sombra acústica y algo menos definida que en $A$, más frecuente de observar en fase reabsortiva. C. Calcificación mal definida, con imágenes puntiformes, también frecuente en fase reabsortiva.

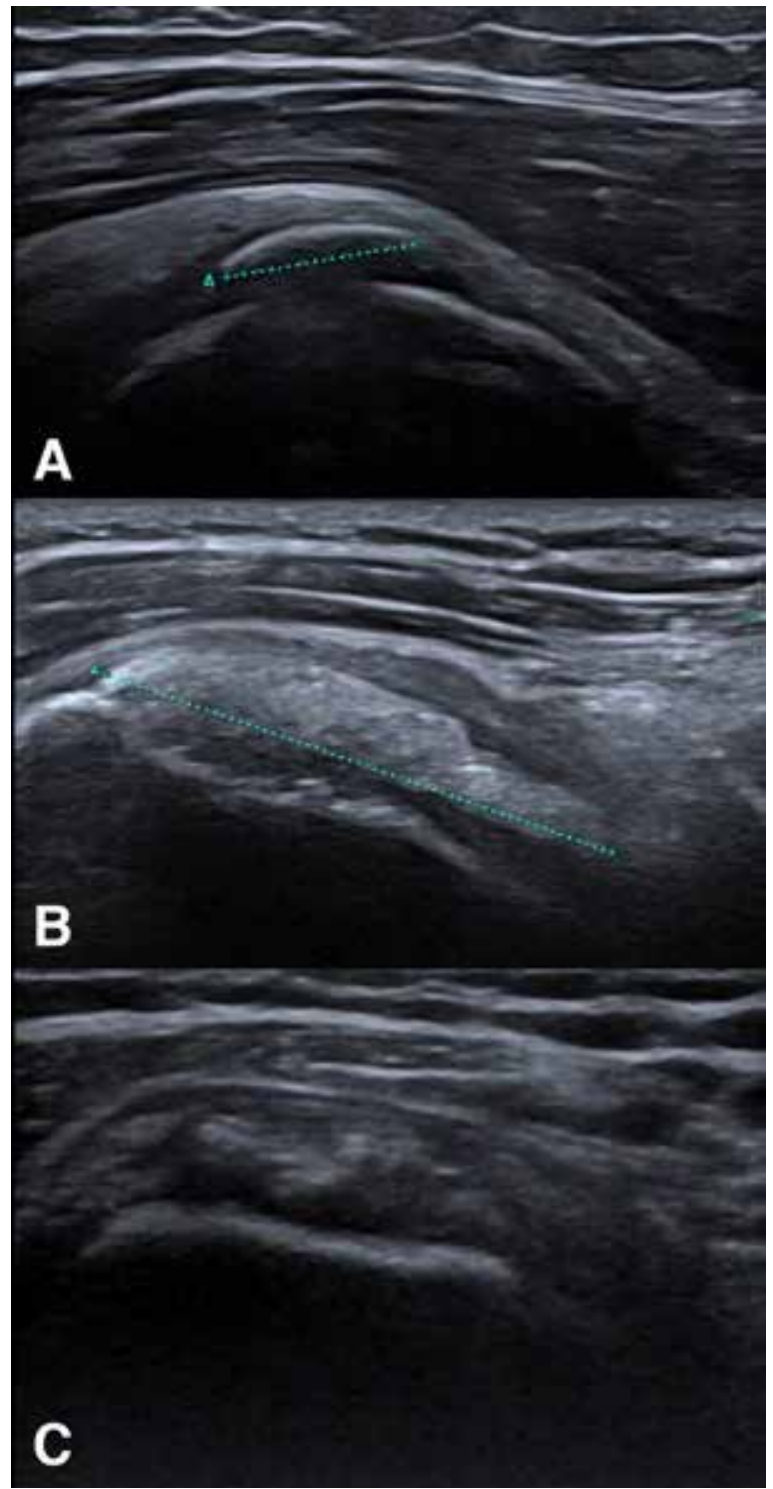




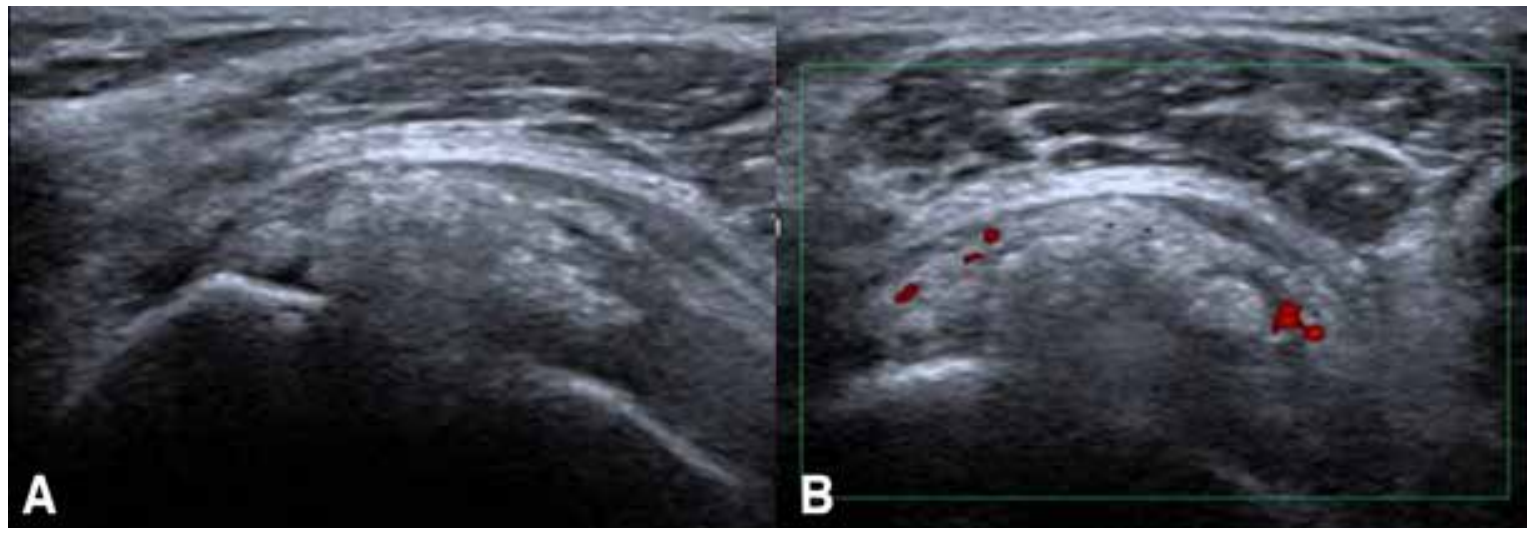

Figura 3: A. Calcificación polimorfa con escasa sombra acústica. B. Hipervascularización al Doppler color, característico en fase reabsortiva.

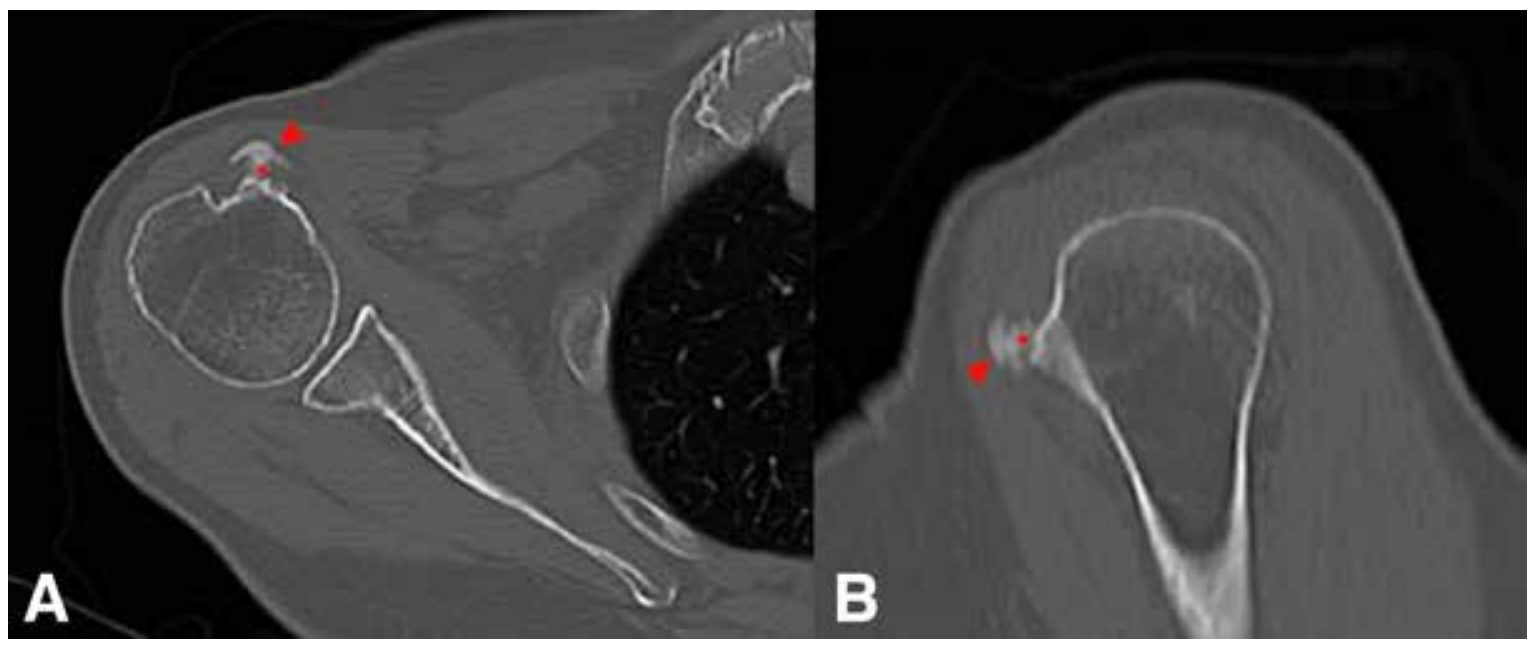

Figura 4: Imagen de TC de hombro. A. Corte axial y B. corte sagital en ventana ósea, muestran calcificación parcialmente definida en el espesor del tendón del subescapular $\left(^{*}\right)$ con extension al plano anterior en la bursa subacromial deltoidea (cabezas de flechas rojas).

\section{Resonancia magnética}

La RM es una herramienta adicional pero no indispensable en el estudio imagenológico de la tendinopatía cálcica. Los depósitos de hidroxiapatita de calcio tienen en general baja señal en todas las secuencias (Figura 5a), aunque en fase reabsortiva pueden observarse áreas de mayor señal en la calcificación (Figura 5b) como manifestación de edema, por lo que podría eventualmente malinterpretarse como lesión tendínea. Además, la RM entrega mayor información del compromiso inflamatorio en las partes blandas que se observa habitualmente en fase reabsortiva y de migración bursal (Figuras 5 b y 6 ), lo cual en localizaciones menos frecuentes puede llevar a errores diagnósticos ya que se pueden presentar con edema óseo (Figura 7) e incluso erosión cortical(28). El rendimiento diagnóstico de la RM es sobre el $95 \%$, aunque disminuye con calcificaciones más pequeñas.
En estos casos, las secuencias gradientes son de mayor utilidad, debido al artefacto de susceptibilidad magnética que produce el calcio(29).

\section{Pitfalls}

Los principales errores diagnósticos en la tendinopatía cálcica se dan en el estudio con RM, en donde se puede malinterpretar como una lesión agresiva (edema óseo, erosión cortical) o patología infecciosa, debido al extenso compromiso inflamatorio de partes blandas, especialmente en localizaciones más allá del manguito rotador (Figura 8). En estos casos, el hallazgo de calcificaciones en el trayecto del tendón, ausencia de derrame articular y presentación clínica aguda orientan a una tendinopatía cálcica, lo que es de suma importancia, ya que se trata de una patología autolimitada y no agresiva. En ese sentido, el complemento radiográfico puede ser de gran ayuda. 


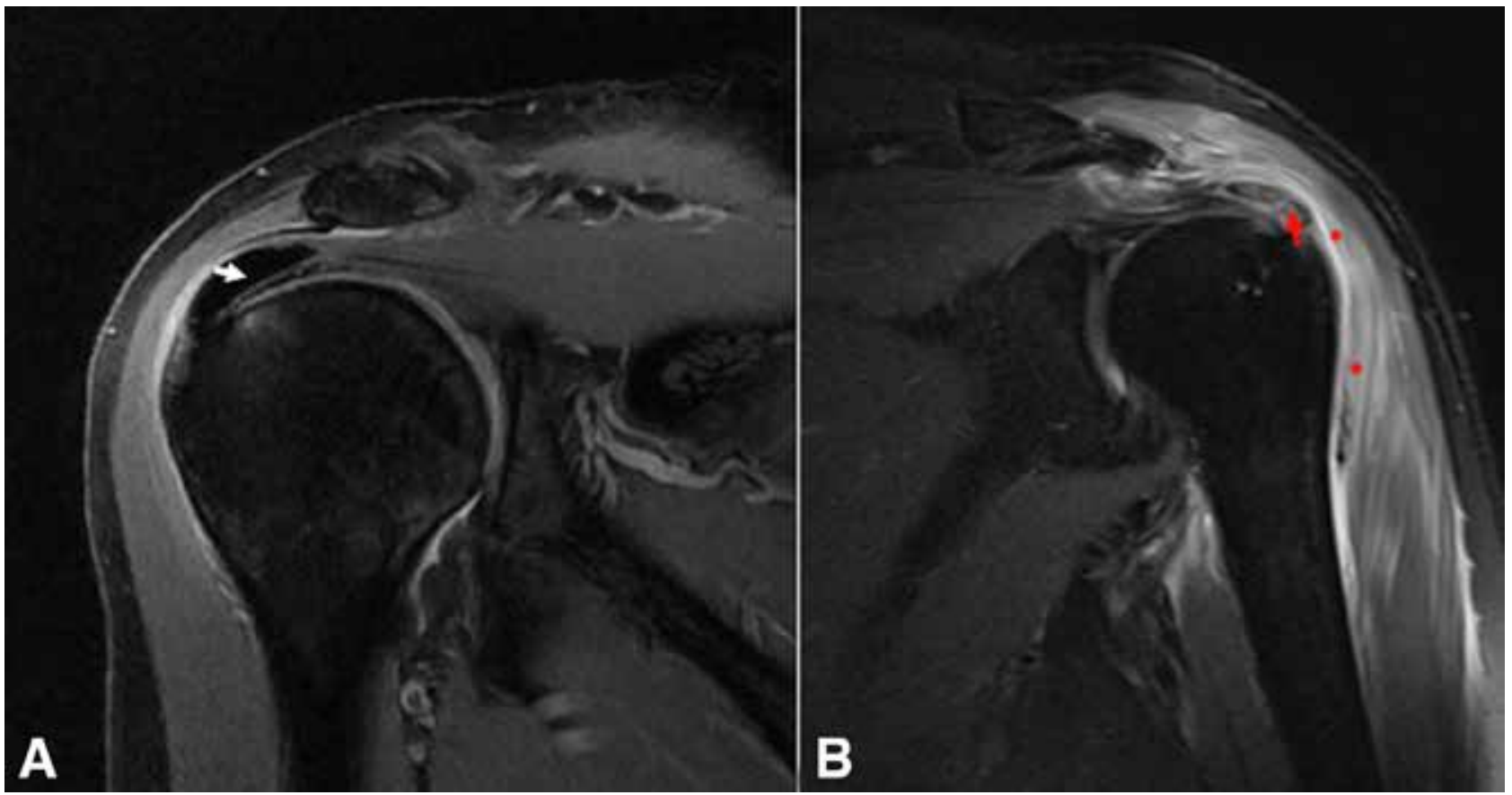

Figura 5: A. Corte DP FS Coronal, gruesa calcificación en el espesor del supraespinoso, de baja señal y bien definida (flecha blanca). B. Corte DP FS Coronal, calcificación en el supraespinoso con algunas áreas de mayor señal (flecha roja), asociado a extenso compromiso inflamatorio de las partes blandas ( ${ }^{*}$ ), en el contexto de una fase reabsortiva.

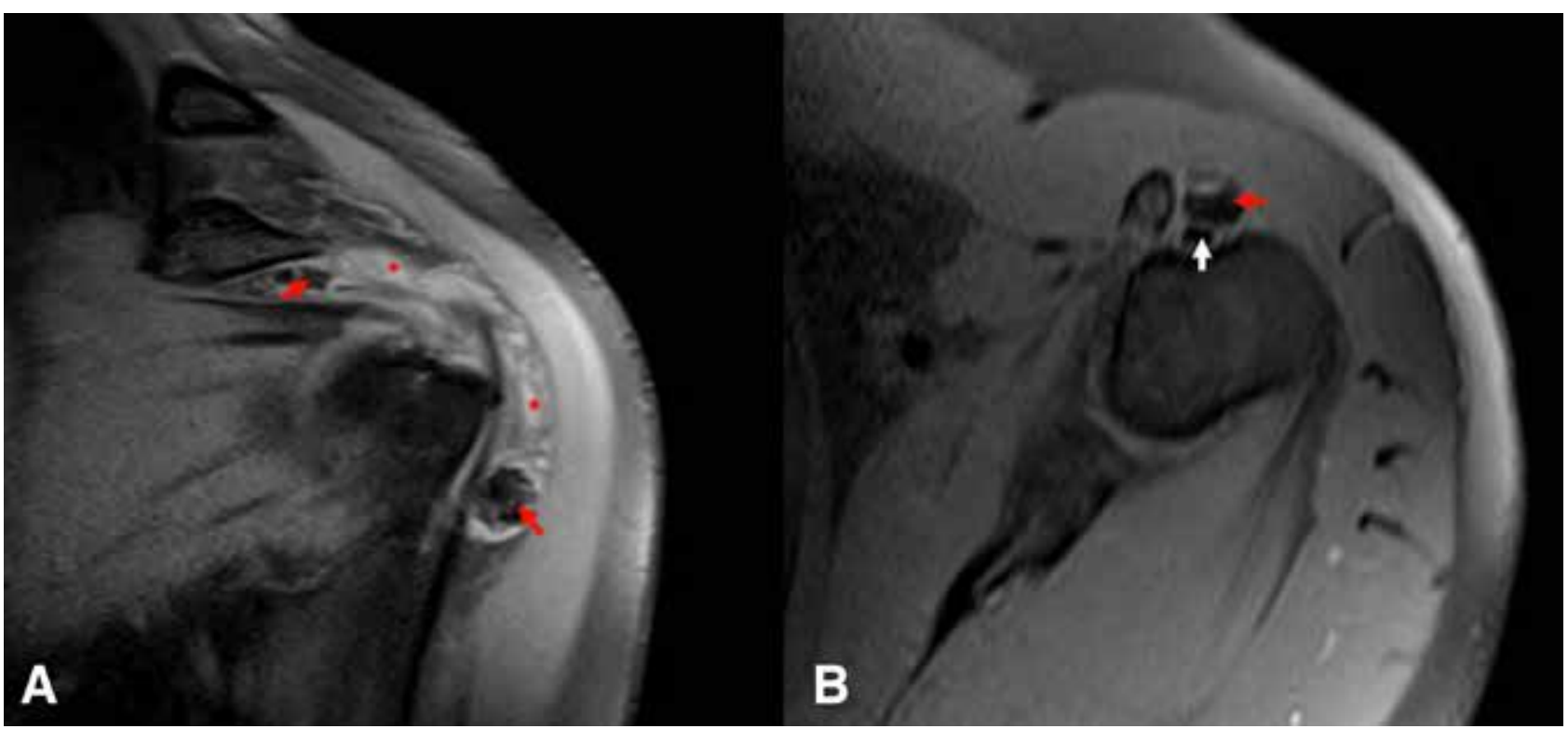

Figura 6: Migración bursal. A, corte DP FS Coronal y B, DP FS Axial, muestran calcificaciones (flechas rojas) en el interior de la bursa subacromial subdeltoidea (asteriscos), la que se encuentra marcadamente distendida. Tendón de la cabeza larga del bíceps braquial (flecha blanca). 


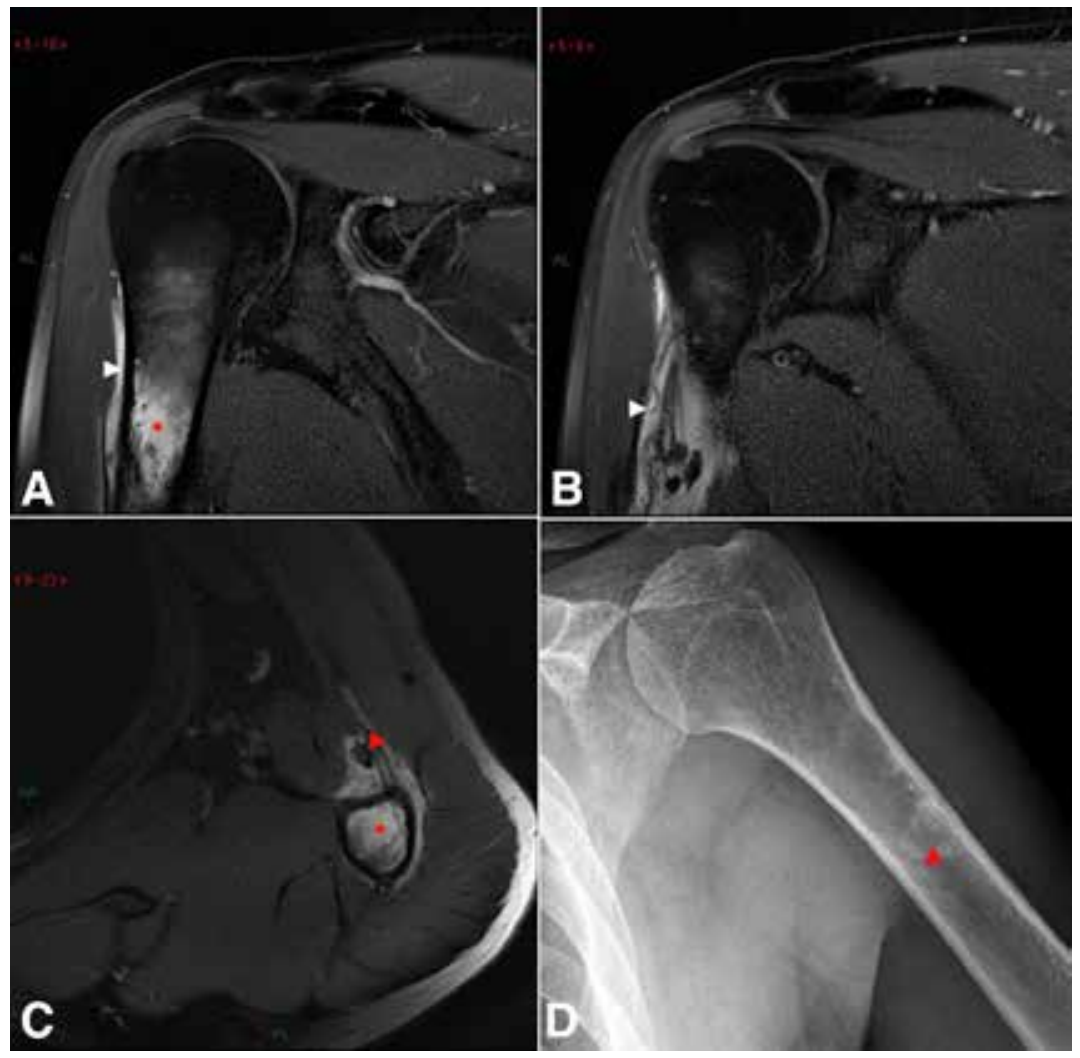

Figura 7: A, B. Cortes DP FS Coronales y C, corte DP FS Axial, muestran intenso edema óseo en el tercio proximal del fémur $\left({ }^{*}\right)$ con signos inflamatorios en las partes blandas adyacentes (cabezas de flecha blancas), en relación a la presencia de calcificaciones en el tendón del pectoral mayor (cabezas de flecha rojas), las que se proyectan en la radiografía en $D$ (cabeza de flecha roja).

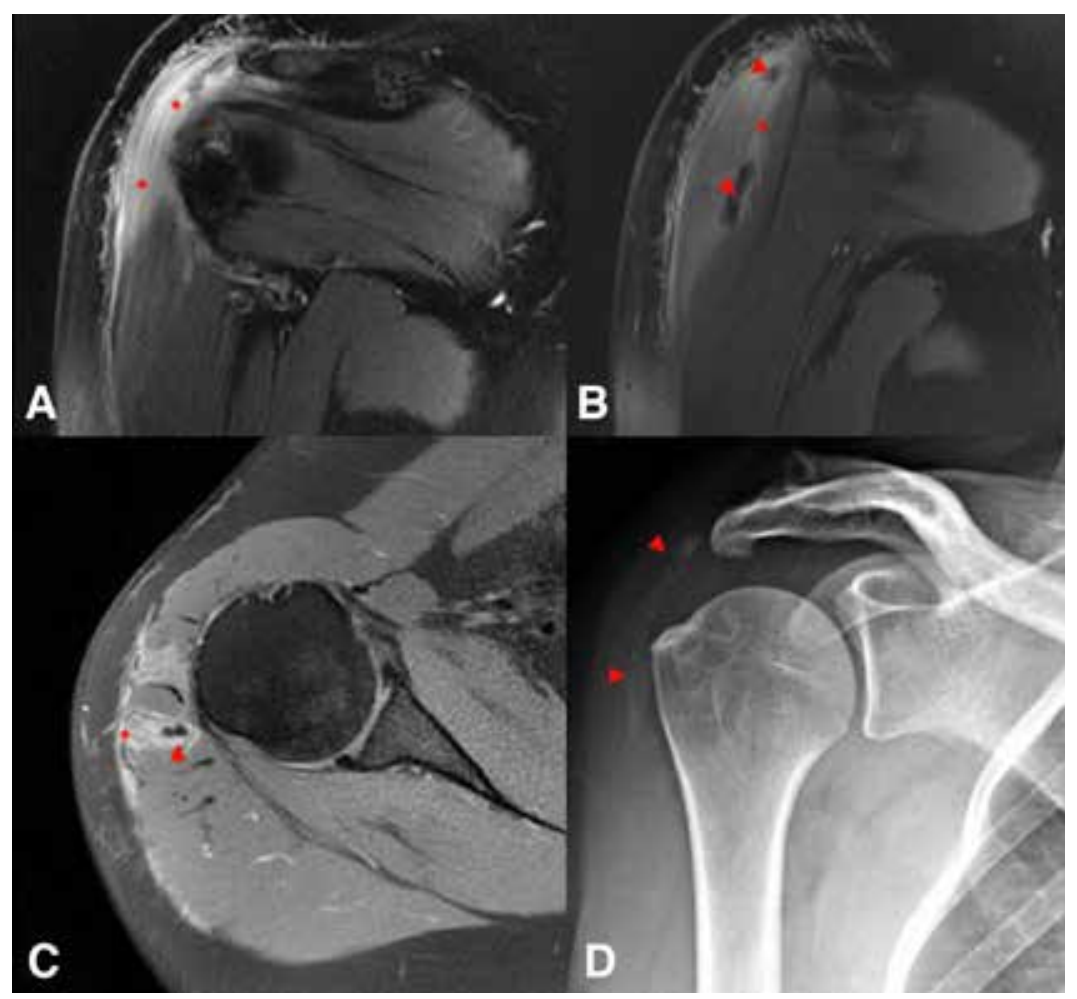

Figura 8: A, B. Cortes sucesivos DP FS Coronales y C, corte DP FS Axial, demuestran amplio compromiso inflamatorio del deltoides $\left(^{*}\right)$, que podría perfectamente malinterpretarse como patología infecciosa. Sin embargo, la presencia de calcificaciones en el trayecto de uno de los fascículos tendíneos del deltoides (cabezas de flecha rojas), evidentes también en radiografía en $D$, hacen el diagnóstico de tendinopatía cálcica. 
Además, si se decide biopsiar, para el patólogo son casos de difícil diagnóstico, ya que la metaplasia cartilaginosa en la tendinopatía cálcica puede ser fácilmente malinterpretada como tumor condroide ${ }^{(26)}$.

Un caso particular de tendinopatía cálcica que puede ser fácilmente malinterpretado es el depósito de cristales de hidroxiapatita en el tendón del músculo largo del cuello (longus colli), en donde los pacientes consultan por intenso dolor, a veces asociado a elevación de parámetros inflamatorios e incluso fiebre. Si se evalúa con TC se observa líquido en el plano del espacio retrofaríngeo, el cual puede interpretarse como colección / absceso que podría tener indicación quirúrgica, la cual no está indicada en la tendinopatía cálcica (Figura 9). En este caso particular, al tratarse de cuadros clínicos muy similares, la interpretación radiológica es de vital importancia, ya que determinará la conducta clínica a seguir (cirugía vs. tratamiento sintomático).
Rol de la ecografía en el manejo terapéutico

La primera línea del manejo de la tendinopatía cálcica es el tratamiento conservador con antiinflamatorios y kinesiterapia. La aspiración y lavado guiado bajo ecografía asociado a la infiltración de corticoides en la bursa subacromial subdeltoidea ha ido ganando espacio y se utiliza cada vez más frecuentemente como parte del manejo inicial debido a su disponibilidad, alivio inmediato del dolor y baja tasa de complicaciones, con buen pronóstico a largo plazo, evitando en la mayoría de los casos la cirugía ${ }^{(30,31)}$. En nuestro centro, es parte del manejo en prácticamente todos los pacientes que se presentan en fase reabsortiva. También se incluye en el manejo de los casos más allá del manguito rotador (Figura 10), con excelentes resultados inmediatos. En aquellos casos refractarios, un segundo procedimiento también ha demostrado ser beneficioso ${ }^{(31)}$.

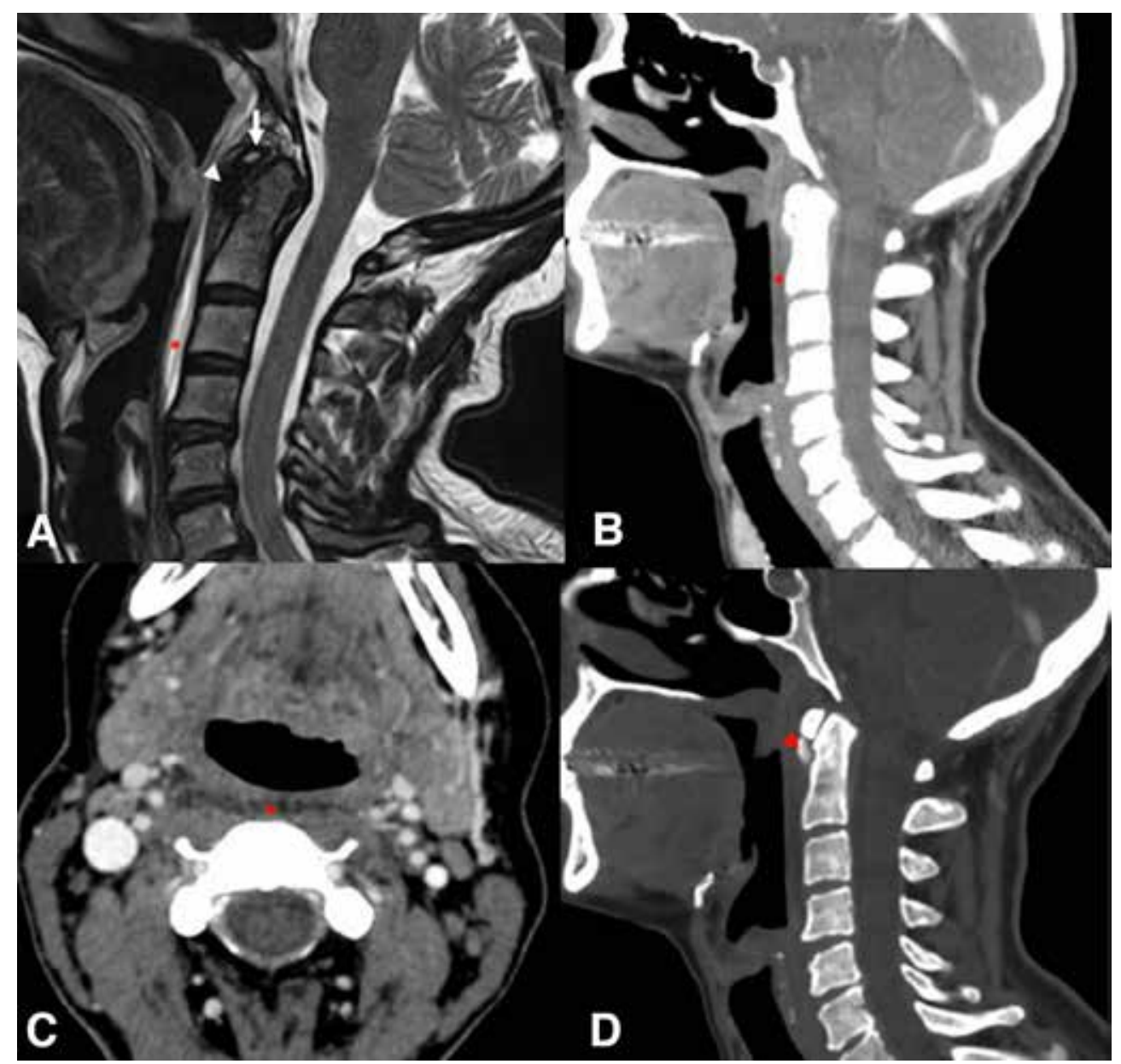

Figura 9: Paciente de 54 años que consultó por cervicalgia. A. En el estudio inicial con RM, se demuestran algunas discopatías degenerativas, pero llama la atención líquida en el espacio retrofaríngeo (*), por lo que se evaluó con TC de cuello $(B, C)$, donde se confirma la presencia de líquido retrofaríngeo (*). En ventana ósea (D), demuestra con mayor claridad las calcificaciones en el tendón del músculo longus colli (cabeza de flecha roja), las que no eran de fácil interpretación en la RM (cabeza de flecha blanca en A), debido a la estrecha relación con el arco anterior del atlas (flecha blanca en A). 


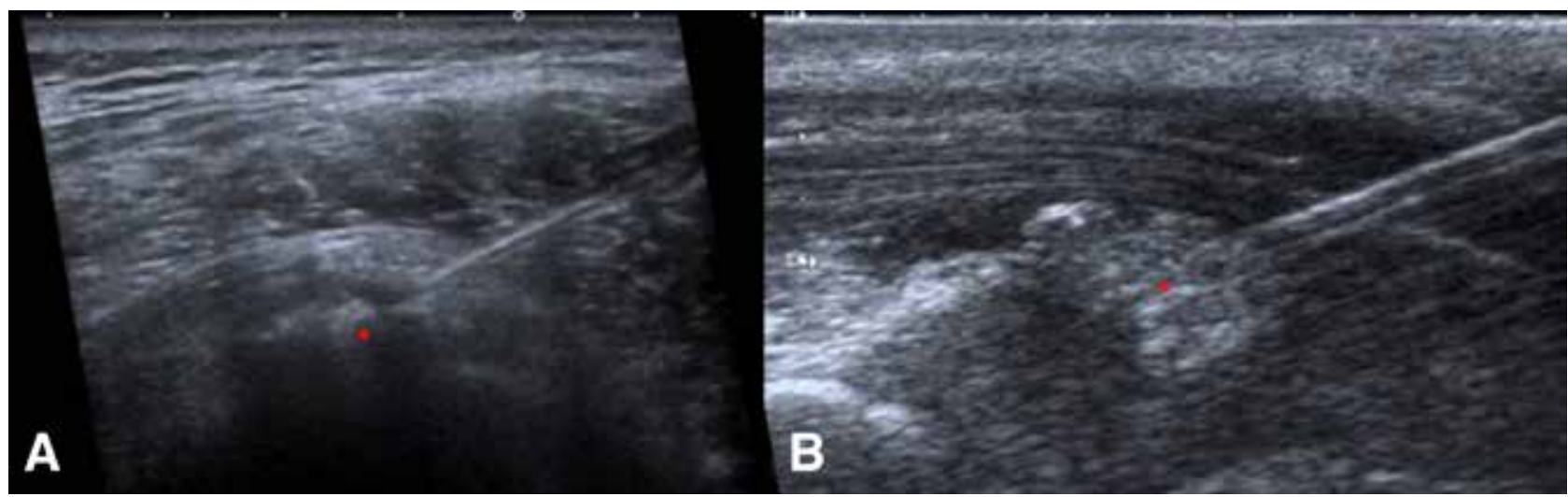

Figura 10: Aspiración con aguja guiada bajo ecografía. A. Calcificación ( ${ }^{*}$ ) en el espesor del glúteo medio visualizada en la radiografía en la figura $1 E$. B. Calcificación $\left({ }^{*}\right)$ en relación a los tendones flexores visualizada en la figura $1 F$.

\section{Conclusión}

La tendinopatía cálcica es el depósito de cristales de hidroxiapatita cálcica en los tendones, es más frecuente de encontrar en los tendondes del manguito rotador, siendo una causa común de hombro doloroso, aunque cualquier tendón puede verse afectado.

Los depósitos cálcicos se generan a partir de una metaplasia de los tenocitos, la cual todavía no está del todo esclarecida. La composición de las calcificaciones y el manejo es distinto al de las calcificaciones distróficas visualizadas en patología degenerativa (calcificaciones entesopáticas), por lo que es importante describirlo y reportarlo como tal en los informes radiológicos para orientar correctamente al clínico.

El estudio imagenológico en la gran mayoría de los casos es suficiente con la radiografía simple y la ecografía; esta última además juega un rol relevante en el manejo, al servir como guía para la aspiración con aguja. En el estudio con RM, el extenso compromiso de partes blandas puede ser malinterpretado como patología agresiva o infecciosa, por lo que hay que tener esta patología en mente, especialmente en aquellos lugares donde es menos frecuente.

\section{Referencias}

1. Codman EA. The shoulder, rupture of the supraspinatus tendon and other lesions in or about the subacromial bursa. Boston, MA. T. Todd Co; 1934.

2. Hayes CW, Conway WF. Calcium hydroxyapatite deposition disease. Radiographics 1990; 10: 1031e48.

3. Bosworth BM. Calcium deposits in the shoulder and subacromial bursitis: a survey of 12,122 shoulders. JAMA 1941; 116(22): 2477-2482.

4. DePalma AF, Kruper JS. Long term study of shoulder joints afflicted and treated for calcific tendinitis. Clin Orthop 1961; 20: 61-72.

5. Merolla G, Singh S, Paladini P, Porcellini G. Calcific tendinitis of the rotator cuff: state of the art in diagnosis and treatment. J Orthop Traumatol 2016 mar; 17(1): 7-14.

6. Speed CA, Hazleman BL. Calcific tendinitis of the shoulder. N Engl J Med 1999; 340: 1582 e4.

7. Wolfgang GL. Surgical repair of tears of the rotator cuff of the shoulder: Factors influencing the result. J Bone Joint Surg Am 1974; 56(1): 14-26.

8. Jim YF, Hsu HC, Chang CY, Wu JJ, Chang T. Coexistence of calcific tendinitis and rotator cuff tear: an arthrographic study. Skeletal Radiol 1993; 22: 183-185.

9. Ark JW, Flock TJ, Flatow EL. Arthroscopic treatment of calcific tendinitis of the shoulder. Arthroscopy 1992; 8: $183 \mathrm{e} 8$.

10. Wainner RS, Hasz M. Management of acute calcific tendinitis of the shoulder. J Orthop Sports Phys Ther 1998; 27: 231e7.

11. Beckmann N, Tran M, Cai C. Incidence of rotator cuff tears in the setting of calcific tendinopathy on MRI: A case controlled comparison. Skeletal Radiol 2019 Feb; 48(2): 245-250.

12. Chiou HJ, Chou YH, Wu JJ, Hsu CC. Evaluation of calcific tendonitis of the rotator cuff: role of color doppler ultrasonography. J Ultrasound Med 2002; 21(3): 289295.

13. Gartner J, Simons B. Analysis of calcific deposits in calcifying tendinitis. Clin Orthop Relat Res 1990; 254: $111 \mathrm{e} 20$.

14. Riley GP, Harrall RL, Constant CR. Prevalence and possible pathological significance of calcium phosphate salt accumulation in tendon matrix degeneration. Ann Rheum Dis 1996; 55: 109e15.

15. Uhthoff HK, Sarkar K, Maynard JA. Calcifying tendinitis: A new concept of its pathogenesis. Clin Orthop Relat Res 1976; 118: 164-168.

16. Rui YF, Lui PP, Chan LS, Chan KM, Fu SC, Li G. Does 
erroneous differentiation of tendon-derived stem cells contribute to the pathogenesis of calcifying tendinopathy? Chin Med J 2011; 124(4): 606-610.

17. Uhthoff HK. Calcifying tendinitis, an active cell-mediated calcification. Virchows Arch A Pathol Anat Histol 1975; 366: 51-58.

18. Hackett L, Millar NL, Lam P, Murrell GA. Are the Symptoms of Calcific Tendinitis Due to Neoinnervation and/ or Neovascularization? J Bone Joint Surg Am 2016 Feb 3; 98(3): 186-192. doi: 10.2106/JBJS.0.00417.

19. Harvie P, Pollard TC, Carr AJ. Calcific tendinitis: natural history and association with endocrine disorders. J Shoulder Elbow Surg 2007; 16(2): 169-173.

20. Mavrikakis ME, Drimis S, Kontoyannis DA, Rasidakis A, Moulopoulou ES, Kontoyannis S. Calcific shoulder periarthritis (tendinitis) in adult onset diabetes mellitus: a controlled study. Ann Rheum Dis 1989; 48(3): 211-214.

21. Maier M, Schmidt-Ramsin J, Glaser C, Kunz A, Küchenhoff $\mathrm{H}$, Tischer $\mathrm{T}$. Intra- and interobserver reliability of classification scores in calcific tendinitis using plain radiographs and CT scans. Acta Orthop Belg 2008; 74(5): 590-595.

22. Papatheodorou A, Ellinas P, Takis F, Tsanis A, Maris I, Batakis N. US of the shoulder: rotator cuff and nonrotator cuff disorders. Radiographics 2006; 26(1): e23.

23. Lin $\mathrm{CH}$, Chao HL, Chiou HJ. Calcified plaque resorptive status as determined by high-resolution ultrasound is predictive of successful conservative management of calcific tendinosis. Eur J Radiol 2012; 81(8): 17761781.

24. Le Goff B, Berthelot JM, Guillot P, Gle'marec J, Maugars $Y$. Assessment of calcific tendonitis of rotator cuff by ultrasonography: comparison between symptomatic and asymptomatic shoulders. Jt Bone Spine 2010; 77(3): 258-263.

25. Chiou HJ, Chou YH, Wu JJ, Hsu CC, Huang DY, Chang CY. Evaluation of calcific tendonitis of the rotator cuff: Role of color Doppler ultrasonography. J Ultrasound Med 2002; 21(3): 289-295.

26. Flemming DJ, Murphey MD, Shekitka KM, et al. Osseous involvement in calcific tendinitis: a retrospective review of 50 cases. AJR Am J Roentgenol 2003; 181: $965 \mathrm{e} 72$.

27. Cahir J, Saifuddin A. Calcific tendonitis of pectoralis major: CT and MRI findings. Skeletal Radiol. 2005 apr; 34(4): 234-238.

28. Chan R, Kim D, Millet P, Weissman BN. Calcifying tendinitis of rotator cuff with cortical bone erosion. Skeletal Radiol 2004; 33(10): 596-599.

29. Zubler C, Mengiardi B, Schmid MR, Hodler J, Jost B, Pfirrmann CW. MR arthrography in calcific tendinitis of the shoulder: diagnostic performance and pitfalls. Eur Radiol 2007; 17(6): 1603-1610.

30. Wu YC, Tsai WC, Tu YK, Yu TY. Comparative Effectiveness of Nonoperative Treatments for Chronic Calcific Tendinitis of the Shoulder: A Systematic Review and Network Meta-Analysis of Randomized Controlled Trials. Arch Phys Med Rehabil. 2017 aug; 98(8): 1678-1692.e6.

31. Oudelaar BW, Schepers-Bok R, Ooms EM, Huis In 't Veld R, Vochteloo AJ. Needle aspiration of calcific deposits (NACD) for calcific tendinitis is safe and effective: Six months follow-up of clinical results and complications in a series of 431 patients. Eur J Radiol 2016 apr; 85(4): 689-694. 